einstein

Official Publication of the Instituto Israelita

de Ensino e Pesquisa Albert Einstein

ISSN: 1679-4508 | e-ISSN: 2317-6385

\title{
Acute pancreatitis and COVID-19: a new target for infection?
}

\author{
Júlia Aith Balthazar ${ }^{1}$, Ethel Zimberg Chehter $^{1}$ \\ 'Centro Universitário FMABC, Santo André, SP, Brazil.
}

DOI: 10.31744/einstein_journal/2022RW6667

\section{ABSTRACT}

This review aimed to investigate whether SARS-CoV-2 is capable of infecting the gland and causing acute pancreatitis, and the peculiarities in the management of these cases. The research was conducted through PubMed ${ }^{\circledR}$ database, and 62 articles were systematically selected for analysis. Differences were found in the literature; however, there are important warnings, such as the presence of hyperlipasemia, clinical and imaging findings suggestive of acute pancreatitis in the presence and even in the absence of respiratory symptoms. Attention should be paid to clinical and imaging findings during this virus infection, since it is possible to identify these two diseases early. Therefore, it is possible to detect and isolate these patients more quickly, providing the correct care and decreasing the morbidity and mortality of two potentially severe diseases.

Keywords: Pancreatitis; Coronavirus infections; COVID-19; SARS-CoV-2; Betacoronavirus

\section{INTRODUCTION}

Already in its third wave of new cases, severe acute respiratory syndrome coronavirus 2 (SARS-CoV-2) has continued for one year keeping the world under a pandemic with more than 2.8 million deaths. ${ }^{(1)}$ The virus was initially detected in December 2019 in Wuhan, China, with atypical findings of viral pneumonia. On March 11, 2020, the virus had spread across the globe, and a pandemic for coronavirus 2019 disease (COVID-19) was determined by the World Health Organization (WHO). ${ }^{(2)}$

As the number of cases increased, extrapulmonary symptoms were found,

How to cite this article:

Balthazar JA, Chehter EZ. Acute pancreatitis and COVID-19: a new target for infection? einstein (São Paulo). 2022;20:eRW6667.

\section{Corresponding author: Júlia Aith Balthazar \\ Avenida Príncipe de Gales, 821, \\ Portaria 1 - Vila Príncipe de Gales \\ Zip code: 09060-650 - Santo André, SP, Brazil \\ Phone: (55 11) 4993-5400 \\ E-mail: juliaith@gmail.com}

\section{Received on:}

Apr 9, 2021

\section{Accepted on:}

July 19, 2021.

\section{Copyright 2022}

\section{(c) BY}

This content is licensed

under a Creative Commons

Attribution 4.0 International License. such as ageusia, anosmia, diarrhea, myocarditis, and urticaria, among others. ${ }^{(3)}$ This was due to the ability of the virus to infect the host cells through the angiotensin-converting enzyme 2 (ACE2) receptor, which is present in various tissues, such as the respiratory tract, cardiovascular system, kidneys, and intestines. ${ }^{(4)}$ Among the gastrointestinal manifestations, an incidence of $3 \%$ to $79 \%$ of symptoms was detected, ranging from vomiting, anorexia, diarrhea, and nausea to gastrointestinal bleeding. ${ }^{(5)}$

Miao et al., reported the first case of acute pancreatitis in a patient with COVID-19 without respiratory symptoms. ${ }^{(6)}$ Along the same line, other studies were published regarding pancreatic involvement by SARS-CoV-2, suggesting that the virus can cause pancreatic injury. ${ }^{(7)}$ Supporting these findings, Liu et al., identified that ACE2 receptor is expressed in the pancreas in healthy people, and is even slightly more significant in pancreatic tissue than in the lungs, proposing that SARS-CoV-2 may cause pancreatic injury by binding to pancreatic ACE2. ${ }^{(8)}$ 
Few pathological studies in patients with COVID-19 have been performed with emphasis on the pancreas to support these hypotheses, but it was possible to find autopsies in the literature describing degeneration of pancreatic cells, ${ }^{(9)}$ interstitial fibrosis, and lipomatosis separating the acinar cells ${ }^{(10)}$ in these patients, besides detecting SARS-CoV-2 RNA in pancreatic cells. ${ }^{(11)}$

Acute pancreatitis is a potentially severe disease, with the main causes being cholelithiasis ( $40 \%$ to $70 \%$ ), and alcoholism (25\% to $35 \%)$. Other rare causes (10\%), such as medications, trauma, endoscopic retrograde cholangiopancreatography (ERCP), hyperkalemia, hypertriglyceridemia $(>1,000 \mathrm{mg} / \mathrm{dL})$, infection, genetics, and autoimmune diseases. ${ }^{(12)}$ Among the infectious agents are viruses (hepatotropic, coxsackievirus, cytomegalovirus (CMV), human immunodeficiency virus (HIV), herpes simplex virus, paramyxovirus, and varicella-zoster virus), bacteria (mycoplasma, Legionella, Salmonella, and leptospira), fungi (Aspergillus), and parasites (toxoplasma, Cryptosporidium, and Ascaris). ${ }^{(13)}$

In the current context, in which several articles described acute pancreatitis in patients who tested positive for COVID-19 infection, it is suggested the virus may be another infectious cause of pancreatic injury, as well as several other agents described here.

\section{OBJECTIVE}

To investigate the capacity of SARS-CoV-2 of causing acute pancreatitis and the peculiarities in diagnosis and care of these cases.

\section{METHODS}

The database chosen was PubMed ${ }^{\circledast}$. On February 16, 2020, articles with the words "COVID-19" and "acute pancreatitis" were selected. From this search, 105 articles were found; one article was added manually. Inclusion criteria were studies that associated acute pancreatitis with COVID-19, as well as those about hyperlipasemia or hyperamylasemia and COVID-19. Exclusion criteria were articles about acute pancreatitis from another well-defined cause; in letter or comment form; with patients with a negative result for COVID-19; about the treatment of pancreatitis; about multisystem inflammatory syndrome of the child; and that did not deal with acute pancreatitis. Figure 1 shows the Preferred Reporting Items for Systematic Reviews and MetaAnalyses (PRISMA) diagram, based on the reading of titles and abstracts; 30 articles were excluded, leaving 74. Of these, 12 were excluded because they dealt with the mechanism of action of the virus, acute pancreatitis with negative COVID-19 results; they either did not mention the disease or pancreatic alterations, or had a defined cause for acute pancreatitis. Sixty-two articles were then included. The initial analysis was done by the main author and later reviewed by the supervisor of this work. Information such as patients' age, comorbidities, and personal history, chief complaint, gastrointestinal symptoms, pneumonia, or severe acute respiratory syndrome (SARS), type of COVID-19 diagnostic test, medications, laboratory and imaging tests, and diagnostic criteria for pancreatitis, whenever cited, were used for analysis in this study. Regarding the diagnostic criteria, the Revised Atlanta Classification of acute pancreatitis, of 2012, was considered standard. Among the radiological findings, localized or diffuse enlargement of the pancreas, blurring of peripancreatic fat, presence of periglandular collections, pancreatic necrosis, and pancreatic pseudocyst were considered late presentations. Regarding the increase in pancreatic enzymes, hyperamylasemia shows high sensitivity and low specificity, whereas hyperlipasemia shows high sensitivity and specificity for the diagnosis of acute pancreatitis.

\section{| RESULTS}

The research findings were divided into three tables, one containing literature reviews, prospective and retrospective studies (Table 1), another with the case reports (Table 2), and the last with the frequencies of the case report findings (Table 3 ).

In 23 studies, including 14 cohort studies, six literature reviews, one case-control study, and two prospective studies, 12 of them concluded there is some relation between COVID-19 and the pancreas or with

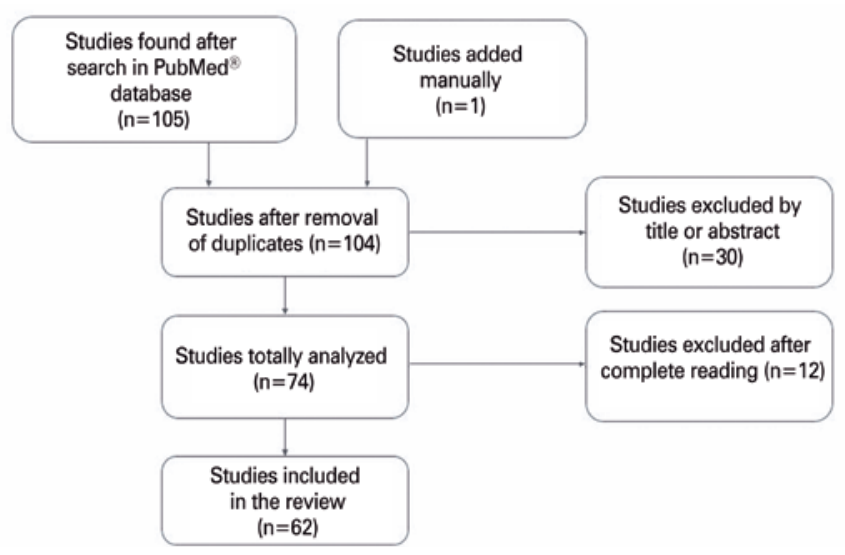

Figure 1. Preferred Reporting Items for Systematic Reviews and Meta-Analyses (PRISMA) diagram 
acute pancreatitis; seven concluded no association but considered some data, and three concluded that there is no association.

Zippi et al., performed a literature review of 14 articles that proposed five theories for pancreatic damage in COVID-19. The first one is the direct damage of the virus, which is able to bind to ACE2 receptors, also expressed in the gastrointestinal tract and in the pancreas; the second one is the increase of pancreatic enzymes by kidney failure and by the lack of elimination of enzymes by the kidneys; the third is their translocation due to altered gastrointestinal permeability; the fourth, due to the use of pancreatic toxic drugs used in the treatment of COVID-19, such as lopinavir, ritonavir, tocilizumab, and baricitinib, among others; and the fifth, due to the cytokine storm caused by SARS-CoV-2, which would attack the pancreas, causing damage to the organ. ${ }^{(7)}$ Along the same line, Hegyi et al., concluded that multiple organ failure that occurs in severe forms of COVID-19 resembles the lipotoxicity in severe acute pancreatitis. ${ }^{(15)}$
Furthermore, they suggest that early supplementation with calcium and albumin helps to reduce lipotoxicity and subsequently counteract the cytokine storm, reducing severe outcomes. ${ }^{(15)}$

Still regarding pathophysiology, Rasch et al., pointed out that hyperlipasemia without typical signs of acute pancreatitis is a frequent finding in patients with COVID-19, due to the impaired microcirculation in severe patients, which would explain the increase in lipase rather than an extrapulmonary finding of the viral infection. ${ }^{(22)}$ Goyal et al., and Rathi et al., also did not recommend associating high lipase levels with severity of pancreatic injury in COVID-19, but hyperlipasemia had a greater association with frequency of gastrointestinal signs and symptoms, a threefold increased risk for poor outcome, intensive care unit (ICU) admission, and mechanical ventilation. ${ }^{(32,33)}$ This relation is not fully elucidated, but Akkus et al., suggested amylase and lipase can be used as indicators of disease activity and prognosis in patients with SARS-CoV-2, and can be included in their routine follow-up. ${ }^{(28)}$

Table 1. Data from prospective, retrospective studies, and literature reviews

\begin{tabular}{|c|c|c|c|c|c|c|}
\hline Studies & Type of article & Patients (n) & $\begin{array}{c}\text { Age } \\
\text { (median) }\end{array}$ & Amylase/lipase (U/L) & Diagnosis of pancreatitis & $\begin{array}{c}\text { Relation between COVID-19 } \\
\text { and acute pancreatitis? }\end{array}$ \\
\hline Zippi et al. ${ }^{(7)}$ & Review & 14 & - & - & - & Yes \\
\hline Suchman et al..14\} & Cohort & 13 & 12 & Amylase and lipase $>3 x$ & INSPPIRE & Yes \\
\hline Hegyi et al..$^{(15)}$ & Meta-analysis & 12 & - & - & - & Yes \\
\hline Akarsu et al. ${ }^{(16)}$ & Prospective study & 316 & 54 & Amylase and lipase $>3 x$ & Modified Atlanta Classification & Yes \\
\hline Gupta $^{(17)}$ & Meta-analysis & 4 & 53 & Amylase and lipase $>3 x$ & - & Yes \\
\hline Juhász et al. ${ }^{(18\rangle}$ & Systematic review & 8 & - & - & Atlanta Classification & No \\
\hline McNabb-Baltar et al. ${ }^{(19)}$ & Cohort & 71 & 64.9 & Lipase $>60$ & Atlanta Classification & No \\
\hline Miró et al. ${ }^{(20)}$ & Case-control & 54 & - & Amylase $>3 x$ & Atlanta Classification & No \\
\hline Lax et al..211) & $\begin{array}{l}\text { Prospective study } \\
\text { (autopsy) }\end{array}$ & 11 & 80.5 & - & - & Yes \\
\hline Rasch et al. ${ }^{[22\}}$ & Cohort & 38 & 68.5 & Mean lipase of 422U/L & Modified Atlanta Classification & No \\
\hline Inamdar et al. ${ }^{.23)}$ & Cohort & 32 & $53.44 \pm 16.60$ & Amylase and lipase $>3 x$ & Atlanta Classification & Yes \\
\hline Dirweesh et al. ${ }^{(24)}$ & Cohort & 14 & $55.2 \pm 14.8$ & - & Atlanta Classification & Yes \\
\hline Hanley et al. ${ }^{(25)}$ & Cohort & 8 & - & - & Autopsy & Yes \\
\hline Szatmary et al. ${ }^{(26)}$ & Cohort & 5 & 42 & Median amylasemia of $149 \mathrm{U} / \mathrm{L}$ & Atlanta Classification & Yes \\
\hline Gubatan et al.(27) & Cohort & 8 & $55.3 \pm 18.7$ & - & - & No \\
\hline Akkus et al. ${ }^{(28)}$ & Cohort & 20 & $55.5 \pm 18.9$ & Mean lipase of $91 \mathrm{U} / \mathrm{L}$ & Atlanta Classification & No \\
\hline Samanta et al..$^{(29)}$ & Systematic review & 5 & - & - & - & No \\
\hline Shiralkar et al..$^{(30)}$ & Cohort & 10 & $45.1 \pm 19.6$ & - & Abdominal CT and MRI & Yes \\
\hline Pandanaboyana et al. ${ }^{(31)}$ & Cohort & 149 & $59.9 \pm 17.2$ & Hyperamylasemia & Modified Atlanta Classification & Yes \\
\hline Goyal et al..$^{(32)}$ & Systematic review & 7 & - & Hyperlipasemia & - & No \\
\hline Rathi et al. ${ }^{(33)}$ & Cohort & 83 & - & Median lipase of 391U/L & - & No \\
\hline McGuinness et al.(34) & Cohort & 650 & 57 & Lipase $>3 x$ & Atlanta Classification & No \\
\hline Wang et al. ${ }^{(35)}$ & Cohort & 52 & $55 \pm 15$ & $\begin{array}{c}\text { Median amylase of } \\
115 \pm 25 \mathrm{U} / \mathrm{L} \\
\text { Median lipase of } 71 \pm 34 \mathrm{U} / \mathrm{L}\end{array}$ & $\begin{array}{l}>90 \mathrm{U} / \mathrm{L} \text { amylase and }>70 \mathrm{U} / \mathrm{L} \\
\text { lipase with pancreatic injury }\end{array}$ & Yes \\
\hline
\end{tabular}


Table 2. Key data from the case reports

\begin{tabular}{|c|c|c|c|c|c|c|c|}
\hline Article data & Miao et al. ${ }^{(6)}$ & Aloysius et al. ${ }^{(36)}$ & \multicolumn{2}{|c|}{ Anand et al. ${ }^{(37)}$} & Hadi et al. ${ }^{(38)}$ & Hadi et al. ${ }^{(38)}$ & Meireles et al. ${ }^{(39)}$ \\
\hline Age & 26 & 36 & \multicolumn{2}{|c|}{59} & 47 & 68 & 36 \\
\hline Sex & Female & Female & \multicolumn{2}{|c|}{ Female } & Female & Female & Female \\
\hline Past history & - & Obesity and anxiety disorder & \multicolumn{2}{|c|}{$\begin{array}{l}\text { Thrombophilia and prior } \\
\text { cholecystectomy }\end{array}$} & - & HTN, hypothyroidism, and osteoporosis & $\begin{array}{l}\text { Post-HELLP syndrome, CKD, } \\
\text { and HTN }\end{array}$ \\
\hline Initial symptoms & $\begin{array}{l}\text { Vomiting, epigastric pain, and } \\
\text { fever }\end{array}$ & $\begin{array}{l}\text { Fever, dry cough, dyspnea, } \\
\text { nausea, vomiting, and diarrhea }\end{array}$ & \multicolumn{2}{|c|}{ Fever, sore throat, and myalgia } & $\begin{array}{l}\text { Fever, headache, anorexia, sore } \\
\text { throat, and dyspnea }\end{array}$ & Epigastric pain and fever & Dry cough, fever, and dyspnea \\
\hline GIT symptoms & Yes & Yes & \multicolumn{2}{|c|}{ Yes } & No & Yes & Yes \\
\hline Abdominal pain & Epigastric & $\begin{array}{l}\text { Band-like epigastric pain } \\
\text { irradiating towards the back }\end{array}$ & \multicolumn{2}{|c|}{ - } & - & Epigastric & Band-like epigastric pain \\
\hline Pneumonia & Yes & Yes & \multicolumn{2}{|c|}{ Yes } & Yes & Yes & Yes \\
\hline SARS & No & Yes & \multicolumn{2}{|c|}{ No } & Yes & Yes & Yes \\
\hline Test to confirm COVID-19 & RT-PCR & RT-PCR & \multicolumn{2}{|c|}{ RT-PCR } & RT-PCR & RT-PCR & $?$ \\
\hline Amylase, U/L & Not done & 325 & \multicolumn{2}{|c|}{ Not done } & $>1,500$ & 934 & 718 \\
\hline Lipase, U/L & 430 & 627 & \multicolumn{2}{|c|}{ Not done } & Not done & Not done & 631 \\
\hline $\mathrm{CRP}, \mathrm{mg} / \mathrm{dL}$ & 1.38 & 1.95 & \multicolumn{2}{|c|}{6.27} & - & 7.7 & 11.9 \\
\hline $\begin{array}{l}\text { Imaging test suggestive of acute } \\
\text { pancreatitis }\end{array}$ & Abdominal CT scan & No & \multicolumn{2}{|c|}{ Abdominal CT scan } & Abdominal ultrasound & No & Abdominal CT scan \\
\hline Triglycerides, mg/dL & Not done & 136 & \multicolumn{2}{|c|}{ Not done } & Normal & Normal & 120 \\
\hline Cholelithiasis or alcoholism & No & No & & & No & No & No \\
\hline Diagnosis of pancreatitis & Imaging, clinical, and laboratory & Modified Atlanta Classification & Suggestive al & minal CT scan & $\begin{array}{l}\text { Modified Glasgow Coma Score } \\
\text { for Acute Pancreatitis ( } 5 \text { points }\end{array}$ & $\begin{array}{l}\text { Modified Glasgow Coma Score for } \\
\text { Acute Pancreatitis (5 points) }\end{array}$ & Imaging, clinical, and laboratory \\
\hline CUM & - & Alprazolam & & & - & $\begin{array}{l}\text { Losartan, levothyroxine, alendronate, } \\
\text { and cyanocobalamin (Vitamina B12) }\end{array}$ & Nifedipine and carvedilol \\
\hline Medication on admission & - & - & & & - & - & - \\
\hline Complications & & NIV & & & $\begin{array}{l}\text { OTI and mechanical ventilation } \\
\text { hemodialysis, and ECMO }\end{array}$ & $\begin{array}{c}\text { OTI and mechanical ventilation, and } \\
\text { hemodialysis }\end{array}$ & - \\
\hline Outcome & Resolution & Resolution & & tion & Not available & Not available & Resolution \\
\hline Article data & Shinohara et al. ${ }^{(40)}$ & Meyers et al & & & leung et al..$^{(42)}$ & Kandasamy et al. ${ }^{(43)}$ & Lakshmanan et al..$^{(44)}$ \\
\hline Age & 58 & 67 & & & 38 & 45 & 68 \\
\hline Sex & Male & Male & & & Male & Female & Male \\
\hline Personal antecedents & HTN & HTN and cholecyst & tomy & & - & $?$ & DM, HTN, and CKD \\
\hline Initial symptoms & Fever and dyspnea & $\begin{array}{r}\text { Epigastric pain, fever, tachy } \\
\text { abdomen }\end{array}$ & rdia, and tight & Fever, intense & $\begin{array}{l}\text { epigastric pain, nausea, and } \\
\text { vomiting }\end{array}$ & $\begin{array}{l}\text { Epigastric pain irradiating towards the } \\
\text { back, nausea, and vomiting }\end{array}$ & Loss of appetite and nausea \\
\hline GIT symptoms & Yes & Yes & & & Yes & Yes & Yes \\
\hline Abdominal pain & - & Epigastric & & & Epigastric & Epigastric irradiating towards the back & Denies \\
\hline Pneumonia & Yes & Yes & & & No & Yes & No \\
\hline SARS & Yes & No & & & No & No & No \\
\hline
\end{tabular}




\section{.... Continuation \\ Table 2. Key data from the case reports}

\begin{tabular}{|c|c|c|c|c|c|}
\hline Article data & Shinohara et al. ${ }^{(40)}$ & Meyers et al. ${ }^{(41)}$ & Cheung et al. ${ }^{(42)}$ & Kandasamy et al. ${ }^{(43)}$ & Lakshmanan et al..$^{(44)}$ \\
\hline Test to confirm COVID-19 & RT-PCR & RT-PCR & RT-PCR & RT-PCR & RT-PCR \\
\hline Amylase, U/L & 383 & Not done & Not done & 364 & 1,030 \\
\hline Lipase, U/L & Not done & 5,295 & $1,219.2$ & 293 & 2,035 \\
\hline $\mathrm{CRP}, \mathrm{mg} / \mathrm{dL}$ & 11.51 & Not done & Not done & Not done & 15.8 \\
\hline $\begin{array}{l}\text { Imaging test suggestive of acute } \\
\text { pancreatitis }\end{array}$ & Abdominal CT scan & Abdominal CT scan & Abdominal CT scan and MRI & Abdominal CT scan & Abdominal CT scan \\
\hline Triglycerides, mg/dL & Not done & Normal & Normal & Not done & Normal \\
\hline Cholelithiasis or alcoholism & No & Mild alcoholism & No & No & No \\
\hline Diagnosis of pancreatitis & Imaging, clinical, and laboratory & Abdominal CT & Abdominal CT & Abdominal CT & Imaging, clinical, and laboratory \\
\hline CUM & - & - & - & - & \\
\hline Medication on admission & $\begin{array}{c}\text { Piperacillin- tazobactam, azithromycin, } \\
\text { favipiravir, nafamostat mesilate, and } \\
\text { methylprednisolone }\end{array}$ & - & - & Empirical antibiotic & Empirical antibiotic \\
\hline Complications & $\mathrm{OTI}$ and ECMO & Not available & - & NIV & - \\
\hline Outcome & Resolution & Not available & Resolution & Resolution & Resolution \\
\hline Article data & Gonzalo-Voltas et al..$^{(45)}$ & Brikman et al..$^{(46)}$ & Narang et al. ${ }^{(47)}$ & Wang et al. ${ }^{(48)}$ & Wang et al. ${ }^{(48)}$ \\
\hline Age & 76 & 61 & 20 & 42 & 35 \\
\hline Sex & Female & Male & Female & Male & Male \\
\hline Past history & Hypercholesterolemia and GERD & - & $\begin{array}{l}\text { Primigesta, 33- week gestation, obesity, } \\
\text { and cholecystectomy }\end{array}$ & - & - \\
\hline Initial symptoms & Epigastric pain and vomiting & Fever, dyspnea, and cough & Dry cough and myalgia & $\begin{array}{l}\text { Nausea and } \\
\text { epigastic pain }\end{array}$ & Abdominal pain, nausea, and vomiting \\
\hline GIT symptoms & Yes & Yes & Yes & Yes & Yes \\
\hline Abdominal pain & Band-like epigastric pain & Diffuse abdominal pain & Epigastric pain irradiating to the back & Epigastric pain irradiating to the back & Epigastric pain irradiating to the back \\
\hline Pneumonia & No & Yes & Yes & Yes & Yes \\
\hline SARS & No & Yes & Yes & Yes & No \\
\hline Test to confirm COVID-19 & RT-PCR & RT-PCR & RT-PCR & RT-PCR & RT-PCR \\
\hline Amylase, U/L & 3,568 & 142 & 1,168 & 132 & Normal \\
\hline Lipase, U/L & Not done & 203 & 859 & 382 & 1,042 \\
\hline $\mathrm{CPR}, \mathrm{mg} / \mathrm{dL}$ & 1.9 & - & - & $>20$ & $>20$ \\
\hline $\begin{array}{l}\text { Imaging test suggestive of acute } \\
\text { pancreatitis }\end{array}$ & Abdominal CT and ultrasound & Abdominal CT & Abdominal CT & - & Abdominal CT \\
\hline Triglycerides, mg/dL & Not done & 281.67 & - & 283.44 & 170.06 \\
\hline Cholelithiasis or alcoholism & No & No & No & No & No \\
\hline Diagnosis of pancreatitis & Imaging, clinical, and laboratory & Modified Atlanta Classification & Presumptive & Ranson & Imaging, clinical, and laboratory \\
\hline CUM & Omeprazole 20mg/day & - & - & - & - \\
\hline
\end{tabular}


...Continuation

Table 2. Key data from the case reports

\begin{tabular}{|c|c|c|c|c|c|}
\hline Article data & Gonzalo-Voltas et al..$^{(45)}$ & Brikman et al. ${ }^{(46)}$ & Narang et al. ${ }^{(47)}$ & Wang et al. . $^{(48)}$ & Wang et al..$^{(48)}$ \\
\hline Medication on admission & $\begin{array}{l}\text { Azithromycin, chloroquine, lopinavir, } \\
\text { and ritonavir }\end{array}$ & $\begin{array}{c}\text { Azithromycin, hydroxychloroquine, } \\
\text { zinc, tocilizumabe, dexamethasone, } \\
\text { enoxaparin, lopinavir- ritonavir, } \\
\text { pantoprazole, ciprofloxacin, and } \\
\text { clindamycin }\end{array}$ & - & - & - \\
\hline Complications & - & NIV & $\begin{array}{l}\text { NIV and premature rupture of membranes, } \\
\text { and preterm birth }\end{array}$ & $\begin{array}{l}\text { Cardiac arrest, OTI and mechanical } \\
\text { ventilation, and hemodialysis }\end{array}$ & - \\
\hline Outcome & Resolution & Resolution & Resolution & Death & Resolution \\
\hline Article data & Tollard et al. ${ }^{(49)}$ & Acherjya et al. ${ }^{(50)}$ & Karimzadeh et al. ${ }^{(51)}$ & Simou et al. ${ }^{(52)}$ & Mazrouei et al. (53) \\
\hline Age & 32 & 57 & 65 & 67 & 24 \\
\hline Sex & Female & Female & Female & $?$ & Male \\
\hline Past history & Morbid obesity and diabetic ketoacidosis & $\begin{array}{l}\text { HTN, type } 2 \text { DM, breast and larynx } \\
\text { cancer, and prior cholecystectomy }\end{array}$ & HTN and asthma & $\begin{array}{l}\text { Type } 2 \text { DM, obesity, and prior } \\
\text { cholecystectomy }\end{array}$ & - \\
\hline Initial symptoms & $\begin{array}{l}\text { Dyspnea, polyuria, polydipsia, and } \\
\text { abdominal pain }\end{array}$ & $\begin{array}{l}\text { High fever, myalgia, anosmia, fatigue, } \\
\text { and arthralgia }\end{array}$ & $\begin{array}{l}\text { Epigastric pain, nausea, chills, and myalgia. On the } 7^{\text {th }} \\
\text { day, the patient presented with dyspnea }\end{array}$ & Fever, dyspnea, myalgia, and arthralgia & $\begin{array}{l}\text { Epigastric pain, nausea, vomiting, and } \\
\text { mild respiratory symptoms }\end{array}$ \\
\hline GIT symptoms & Yes & Yes & Yes & No & Yes \\
\hline Abdominal pain & Abdominal & Epigastric pain irradiating to the back & Epigastric & No & Epigastric \\
\hline Pneumonia & Yes & Yes & Yes & Yes & No \\
\hline SARS & & No & No & No & No \\
\hline Test to confirm COVID-19 & RT-PCR & RT-PCR & RT-PCR & RT-PCR & RT-PCR \\
\hline Amylase, U/L & Not done & Not done & 192 & - & 391 \\
\hline Lipase, U/L & 321 & 8,352 & 283 & 576 & 578 \\
\hline $\mathrm{CRP}, \mathrm{mg} / \mathrm{dL}$ & - & 25.2 & - & 4.13 & - \\
\hline $\begin{array}{l}\text { Imaging test suggestive of } \\
\text { acute pancreatitis }\end{array}$ & Abdominal CT scan & Abdominal CT scan & Abdominal CT scan & Abdominal CT scan & Abdominal CT scan \\
\hline Triglycerides, mg/dL & Normal & 276 & 80 & 212.58 & Not done \\
\hline Cholelithiasis or alcoholism & No & No & No & No & No \\
\hline Diagnosis of pancreatitis & Imaging, clinical, and laboratory & Atlanta Classification & Clinical & $\begin{array}{l}\text { Balthazar Classification of Abdominal } \\
\text { CT }\end{array}$ & Atlanta Classification \\
\hline CUM & - & $\begin{array}{l}\text { Radiation therapy, trastuzumab, } \\
\text { losartan, metformin, and insulin }\end{array}$ & - & - & - \\
\hline Medication on admission & - & $\begin{array}{l}\text { Favipiravir and enoxaparin in } \\
\text { prophylactic dose }\end{array}$ & $\begin{array}{l}\text { Levofloxacin, ondansetron, oseltamivir, } \\
\text { hydroxychloroquine, ribavirin, lopinavir, ritonavir, } \\
\text { vancomycin, cefepime, and oxygen therapy }\end{array}$ & $\begin{array}{l}\text { Oxygen therapy, hydroxychloroquine, } \\
\text { azithromycin, methylprednisolone, } \\
\text { vitamin C, zinc, and enoxaparin }\end{array}$ & - \\
\hline Complications & $\begin{array}{l}\text { Diabetic ketoacidosis, pulmonary } \\
\text { thromboembolism, OTI and mechanical } \\
\text { ventilation, and distributive shock }\end{array}$ & NIV & NIV & NIV & - \\
\hline Outcome & Death & Resolution & Resolution & Death & Resolution \\
\hline
\end{tabular}




\section{.... Continuation}

\section{Table 2. Key data from the case reports}

\begin{tabular}{|c|c|c|c|c|c|c|}
\hline Article data & Kumaran et al.(54) & \multicolumn{2}{|c|}{ Kataria et al. (55) } & & AlHarmi et al..$^{(56)}$ & Alwaeli et al. . 57$)$ \\
\hline Age & 67 & \multicolumn{2}{|c|}{46} & & 52 & 30 \\
\hline Sex & Female & \multicolumn{2}{|c|}{ Female } & & Female & Male \\
\hline Past history & $\begin{array}{c}\text { Laparotomy with intestinal resection for stenosis o } \\
\text { the mesenteric artery, and secondary prophylaxis o } \\
\text { thrombosis }\end{array}$ & \multicolumn{2}{|l|}{ of } & Type $2 \mathrm{D}$ & A, HTN, hypothyroidism, and morbid obesity & - \\
\hline Initial symptoms & Epigastric pain, diarrhea, and vomiting & \multicolumn{2}{|c|}{ Fever, dry cough, and dyspnea } & & Fever, dry cough, and dyspnea & $\begin{array}{c}\text { Fever, dry cough, nausea, vomiting, abdominal pain, } \\
\text { diarrhea, and progressive dyspnea }\end{array}$ \\
\hline GIT symptoms & Yes & \multicolumn{2}{|c|}{ Yes } & & Yes & Yes \\
\hline Abdominal pain & Epigastric & \multicolumn{2}{|c|}{$\begin{array}{l}\text { Epigastric pain irradiating } \\
\text { to the back }\end{array}$} & & Abdominal & Epigastric pain irradiating to the back \\
\hline Pneumonia & No & \multicolumn{2}{|c|}{ Yes } & & Yes & Yes \\
\hline SARS & No & \multicolumn{2}{|c|}{ Yes } & & Yes & Yes \\
\hline Test to confirm COVID-19 & RT-PCR & \multicolumn{2}{|c|}{ RT-PCR } & & RT-PCR & RT-PCR \\
\hline Amylase, U/L & 1,483 & \multicolumn{2}{|c|}{501} & & 47 & 151 \\
\hline Lipase, U/L & Not done & \multicolumn{2}{|c|}{1,541} & & & 1,022 \\
\hline $\mathrm{CPR}, \mathrm{mg} / \mathrm{dL}$ & 15.8 & \multicolumn{2}{|c|}{2.51} & & 1.09 & - \\
\hline $\begin{array}{l}\text { Imaging test suggestive of acute } \\
\text { pancreatitis }\end{array}$ & Abdominal CT & \multicolumn{2}{|c|}{ Abdominal CT } & & Abdominal CT & Abdominal CT \\
\hline Triglycerides, mg/dL & 310 & \multicolumn{2}{|c|}{153} & & 168.3 & 133 \\
\hline Cholelithiasis or alcoholism & No & \multicolumn{2}{|c|}{ No } & & No & No \\
\hline Diagnosis of pancreatitis & Modified Atlanta Classification & \multicolumn{2}{|c|}{ Imaging, clinical, and laboratory } & & Imaging and clinical & Imaging, clinical, and laboratory \\
\hline CUM & - & \multicolumn{2}{|c|}{-} & & - & - \\
\hline Medication on admission & Meropenem, metronidazole, and clyndamycin & \multicolumn{2}{|c|}{ Azithromycin, ceftriaxone, and oxygen therapy } & $\begin{array}{r}\text { Dexam } \\
\text { doxycycl } \\
\text { fluticasor }\end{array}$ & $\begin{array}{l}\text { thasone, methylprednisolone, ceftriaxone, } \\
\text { e, azithromycin, enoxaparin, vitamin D, zinc, } \\
\text { e, salbutamol, ipratropium, and pantoprazole }\end{array}$ & $\begin{array}{l}c_{c}, \\
\text { le }\end{array}$ \\
\hline Complications & NIV & \multicolumn{2}{|c|}{ NIV } & & NIV & OTI and mechanical ventilation \\
\hline Outcome & Resolution & \multicolumn{2}{|c|}{ Resolution } & & Resolution & Resolution \\
\hline Article data & Alves et al. ${ }^{(58)}$ & Fernandes et al. ${ }^{(59)}$ & Purayil et al & & Patnaik et al. ${ }^{(61)}$ & Rabice et al. ${ }^{(62)}$ \\
\hline Age & 56 & 36 & 58 & & 29 & 36 \\
\hline Sex & Female & Female & Male & & Male & Female \\
\hline Past history & & - & - & & - & $\begin{array}{c}\text { G4PC2 33-week gestation, obesity, pre-eclampsia, type } 1 \\
\text { DM, and prior cholecystectomy }\end{array}$ \\
\hline Initial symptoms & $\begin{array}{l}\text { Dry cough, dyspnea, malaise, and } \\
\text { abdominal pain }\end{array}$ & ever, headache, and dyspnea & Fever, vomiting, and ep & jastric pain & $\begin{array}{l}\text { Diffuse abdominal pain, irradiating to } \\
\text { the back, fever, and dyspnea }\end{array}$ & Cough and fever \\
\hline GIT symptoms & Yes & Yes & Yes & & Yes & Yes \\
\hline Abdominal pain & Epigastric & Epigastric & Epigastric & & Diffuse, irradiating to the back & Abdominal \\
\hline Pneumonia & Yes & Yes & Yes & & Yes & Yes \\
\hline SARS & Yes & No & No & & & No \\
\hline
\end{tabular}


...Continuation

Table 2. Key data from the case reports

\begin{tabular}{|c|c|c|c|c|c|}
\hline Article data & Alves et al. ${ }^{(58)}$ & Fernandes et al. (59) & Purayil et al. ${ }^{(60)}$ & Patnaik et al. ${ }^{(61)}$ & Rabice et al. ${ }^{(62)}$ \\
\hline Test to confirm COVID-19 & RT-PCR & RT-PCR & RT-PCR & RT-PCR & RT-PCR \\
\hline Amylase, U/L & 544 & 710 & 249 & 2,861 & 88 \\
\hline Lipase, U/L & 2,993 & 640 & $>600$ & 1,650 & 875 \\
\hline $\mathrm{CRP}, \mathrm{mg} / \mathrm{dL}$ & - & - & 29 & 14.6 & - \\
\hline $\begin{array}{l}\text { Imaging test suggestive of acute } \\
\text { pancreatitis }\end{array}$ & Abdominal CT & Abdominal CT & No & Abdominal CT & No \\
\hline Triglycerides, mg/dL & Not done & Not done & Normal & 84 & 210 \\
\hline Cholelithiasis or alcoholism & No & No & No & No & No \\
\hline
\end{tabular}

Diagnosis of pancreatitis

Imaging, clinical, and laboratory

Imaging, clinical, and laboratory

Clinical and laboratory

Imaging, clinical, and laboratory

Clinical and laboratory

Medication on admission

Azithromycin and

hydroxychloroquine

Meropenem and support

Dicloxacillin

Complications OTI and mechanical ventilation

craper

IV and Caesarean section

\section{Outcome}

Resolution

Resolution

Resolution

Resolution

\section{Article data}

Bokhari et al. ${ }^{(63)}$

Samies et al. ${ }^{(64)}$

Samies et al. ${ }^{(64)}$

Samies et al. ${ }^{(64)}$

Kurihara et al. ${ }^{(65)}$

Age 32

Past history

Male

15

Male

16

55

Initial symptoms

Fever, sore throat, productive cough, Vomiting, epigastric pain, fever,

Overweight

Female

15

Prior pancreatitis

\begin{tabular}{lcccc} 
Initial symptoms & $\begin{array}{c}\text { Fever, sore throat, productive cough, } \\
\text { myalgia, and diarrhea }\end{array}$ & $\begin{array}{c}\text { Vomiting, epigastric pain, fever, } \\
\text { ageusia, and anosmia }\end{array}$ & $\begin{array}{c}\text { Abdominal pain, headache, chills, } \\
\text { intermittent hematochezia, and epistaxis }\end{array}$ & Nausea and epigastric abdominal pain \\
\hline GIT symptoms & Yes & Yes & Yes & Pneumonia
\end{tabular}

Abdominal pain

pigastric irradiated to the back Epigastric

Yes

SARS

Yes

Yes

Test to confirm COVID-19

$\begin{array}{ll}\text { No } & \text { RT-PCR } \\ \text { RT-PCR } & \text { Not }\end{array}$

Amylase, U/L

Lipase, U/L

$\mathrm{CPR}, \mathrm{mg} / \mathrm{dL}$

Imaging tests suggestive of

$672 \quad$ Not done

acute pancreatitis

Triglycerides, mg/dL

Cholelithiasis or alcoholism

Not done

Abdominal CT Abdominal CT

riumbilical

Yes

Ro

1.47

953

24.11

Abdominal CT

$\begin{array}{cc}\text { No } & \text { Yes } \\ \text { No } & \text { Yes } \\ \text { RT-PCR } & \text { RT-PCR } \\ \text { Not done } & 252 \\ 1,909 & 263 \\ \text { Not done } & 8.53 \\ \text { minal ultrasound } & \text { Abdominal C }\end{array}$

Diagnosis of pancreatitis

$150 \quad$ Not done Not done

No

No

Not done

Not done
Yes

185

Imaging, clinical, and laboratory Imaging, clinical, and laboratory Clinical and laboratory

Clinical and laboratory

Imaging, clinical, and laboratory

CUM

Medication on admission

Piperacillin-tazobactam

Complications

Acute appendicitis

Lopinavir-ritonavir, azithromycin, and ceftriaxone

Outcome

Resolution

Resolution

Resolution

$\mathrm{OTI}$ and mechanical ventilation, ECMO, and hemodialysis

Resolution Resolution 


\section{.... Continuatio}

Table 2. Key data from the case reports

\begin{tabular}{|c|c|c|c|c|c|}
\hline Article data & Bineshfar et al.(66) & Hassani et al. ${ }^{(67)}$ & Bouali et al. ${ }^{(68)}$ & Schepis et al. ${ }^{(69)}$ & Ahmed et al. ${ }^{(70)}$ \\
\hline Age & 14 & 78 & 60 & 67 & 47 \\
\hline Sex & Male & Female & Female & Female & Male \\
\hline Past history & - & HTN and ischemic heart disease & - & - & - \\
\hline Initial symptoms & Abdominal pain, nausea, and vomiting & Positional epigastric pain, nausea, and vomiting & $\begin{array}{c}\text { Respiratory failure, diffuse abdominal pain, } \\
\text { hematemesis, and melena }\end{array}$ & Fever, epigastric pain, and vomiting & $\begin{array}{c}\text { Fever, sore throat, left-sided cervical edema, } \\
\text { fatigue, and myalgia }\end{array}$ \\
\hline GIT symptoms & Yes & Yes & Yes & Yes & Yes \\
\hline Abdominal pain & Abdominal & Positional epigastric & Diffuse & Epigastric & Diffuse/RIF \\
\hline Pneumonia & No & Yes & No & Yes & Yes \\
\hline SARS & No & Yes & No & No & No \\
\hline Test to confirm COVID-19 & RT-PCR & RT-PCR & RT-PCR & RT-PCR & RT-PCR \\
\hline Amylase, U/L & 1,914 & 1,200 & - & Normal & 349 \\
\hline Lipase, U/L & Not done & 1,450 & 627 & Normal & $>600$ \\
\hline $\mathrm{CRP}, \mathrm{mg} / \mathrm{dL}$ & 4 & - & 8 & Increased & 2.51 \\
\hline $\begin{array}{l}\text { Imaging test suggestive of } \\
\text { acute pancreatitis }\end{array}$ & Abdominal CT & Abdominal ultrasound & Abdominal CT & Abdominal CT & Abdominal CT \\
\hline Triglycerides, mg/dL & Not done & Normal & Not done & Not done & Not done \\
\hline Cholelithiasis or alcoholism & No & No & No & No & No \\
\hline Diagnosis of pancreatitis & Atlanta Classification & Imaging, clinical, and laboratory & $\begin{array}{l}\text { Balthazar Classification } \\
\text { of abdominal CT }\end{array}$ & $\begin{array}{l}\text { Analysis of pancreatic pseudocyst fluid } \\
\text { containing SARS-CoV-2 RNA }\end{array}$ & Clinical and laboratory \\
\hline CUM & - & Valsartan, clopidogrel, ASA, and atorvastatin & - & - & - \\
\hline Medication on admission & - & Remdesivir and interferon beta- $1 \mathrm{~b}$ & - & - & $\begin{array}{l}\text { Hydroxychloroquine, azithromycin, and } \\
\text { cefuroxime }\end{array}$ \\
\hline Complications & - & $\begin{array}{c}\text { OTI and mechanical ventilation, kidney failure, } \\
\text { and cardiorespiratory arrest }\end{array}$ & $\begin{array}{l}\text { Laparotomy, gastrectomy, and } \\
\text { cardiorespiratory arrest }\end{array}$ & Drainage of pancreatic pseudocyst & - \\
\hline Outcome & Resolution & Death & Death & Resolution & Resolution \\
\hline Article data & & \multicolumn{2}{|l|}{ Dietrich et al. ${ }^{(71)}$} & \multicolumn{2}{|c|}{ Chivato et al. ${ }^{(72)}$} \\
\hline Age & & \multicolumn{2}{|l|}{72} & \multicolumn{2}{|l|}{55} \\
\hline Sex & & Male & & \multicolumn{2}{|l|}{ Male } \\
\hline Past history & & \multicolumn{2}{|l|}{ Overweight and HTN } & \multicolumn{2}{|l|}{ - } \\
\hline Initial symptoms & & \multicolumn{2}{|l|}{ Nausea and mild abdominal pain } & \multicolumn{2}{|c|}{ Respiratory failure } \\
\hline GIT symptoms & & Yes & & - & \\
\hline Abdominal pain & & Mild & & - & \\
\hline Pneumonia & & Yes & & Yes & \\
\hline SARS & & Yes & & Yes & \\
\hline Test to confirm COVID-19 & & RT-PCR & & RT-PCR & \\
\hline Amylase, U/L & & Not done & & Increase & \\
\hline
\end{tabular}


Table 2. Key data from the case reports

\begin{tabular}{|c|c|}
\hline Article data & Dietrich et al. ${ }^{(71)}$ \\
\hline Lipase, U/L & 185 \\
\hline CRP, mg/dL & 2.3 \\
\hline Imaging test suggestive of acute pancreatitis & Abdominal ultrasound \\
\hline Triglycerides, mg/dL & Not done \\
\hline Cholelithiasis or alcoholism & Cholelithiasis, without cholestasis \\
\hline Diagnosis of pancreatitis & Imaging, clinical, and laboratory \\
\hline CUM & Beta-blocker \\
\hline Medication on admission & Ceftriaxone and clarithromycin \\
\hline Complications & OTI and mechanical ventilation \\
\hline Outcome & Resolution \\
\hline \multicolumn{2}{|c|}{$\begin{array}{l}\text { HTN: hypertension; HELLP: hemolysis, elevated liver enzymes, low platelet count; CKD: chronic kidney disease; GIT: gastrointestinal tract; SARS: severe acute respirar } \\
\text { intubation; ECMO: extracorporeal membrane oxygenation; DM: diabetes mellitus; MRI: magnetic resonance imaging; GERD: gastroesophageal reflux disease; ASA: ac }\end{array}$} \\
\hline \multicolumn{2}{|c|}{ Table 3. Frequencies of data from the case reports } \\
\hline \multicolumn{2}{|r|}{ Mean 46.47 and median 47} \\
\hline Sex & 22 female, 19 male, and one unknown \\
\hline Comorbidities, \% & 47.62 \\
\hline Abdominal pain, \% & 83.33 \\
\hline Pneumonia, \% & 80.95 \\
\hline SARS, $\%$ & 38.1 \\
\hline Amylase mean, U/L & 779.25 \\
\hline Lipase, U/L & $1,230.882$ \\
\hline C-reactive protein mean, $\mathrm{mg} / \mathrm{dL}$ & 9.73 \\
\hline Imaging suggestive of pancreatitis, $\%$ & 85.71 \\
\hline Diagnostic classification as per Atlanta, \% & $73.8(31 / 42)$ \\
\hline Complications, \%* & 52.38 \\
\hline Outcome, \%* & 80.95 in recovery and 11.9 deaths \\
\hline
\end{tabular}

* Percentage excluding articles that do not mention complications or outcome.

SARS: severe acute respiratory syndrome.

Wang et al., evaluated 52 patients admitted to Zhongnan Hospital of Wuhan University, from January to February 2020, and analyzed pancreatic injury as elevated lipase (>70U/L) and amylase (>90U/L). They identified a pattern of mild pancreatitis present in patients with COVID-19 pneumonia, but it may not be the result of direct viral involvement of pancreatic cells, since there was usually no positive clinical finding for acute pancreatitis. ${ }^{(35)}$

Contrary to the studies described, McNabb-Baltar et al., showed hyperlipasemia ( $>60 \mathrm{U} / \mathrm{L}$ ) was not associated with severe forms of COVID-19 nor with worse clinical outcomes. Furthermore, they did not associate this finding with acute pancreatitis, since $48 \%$ of patients presented with hyperlipasemia, and none had criteria - whether laboratory or tomographic for acute pancreatitis. ${ }^{(19)}$

Two of the included studies diagnosed pancreatitis at autopsy examination. Lax et al., analyzed 11 bodies from patients infected with COVID-19, aged between 66 and 91 years. Acute pancreatitis was identified in four out of 11 patients, and in one third of them, no typical symptoms had been seen. (21) The other study was performed by Hanley et al., with bodies from eight SARS-CoV-2 positive patients, and $25 \%$ of them had acute pancreatitis. Among them, only one patient had microscopic findings of acute pancreatitis. As a limitation, the authors of the latter study could not confirm whether the findings of acute pancreatitis were due to iatrogenesis, comorbidities, or secondary infection. ${ }^{(25)}$

Gubatan et al., in a cohort of eight patients, concluded that those with a history of pancreatitis were more susceptible to COVID-19; $7.8 \%$ of patients with prior pancreatitis had positive COVID-19 serology, whereas $2.8 \%$ of those had no prior pancreatitis. However, the authors did not associate an increased risk of pancreatic inflammation with SARS-CoV-2 infection, since none of the patients in the study had acute pancreatitis during viral infection. ${ }^{(27)}$ 
Idiopathic acute pancreatitis in the setting of COVID-19 has been identified in several studies. Inamdar et al., in a cohort of 32 patients infected with SARS-CoV-2 and diagnosed with acute pancreatitis by the Atlanta Classification, found the idiopathic form of pancreatitis to be the most common. Furthermore, they identified that Hispanic patients with acute pancreatitis were more likely to be diagnosed with COVID-19 than other ethnicities. ${ }^{(23)}$ Corroborating these findings, Szatmary et al., concluded the endocrine pancreas is more vulnerable to COVID-19 infection, and male sex, abdominal pain, metabolic stress, and tomographic findings of pancreatic and duodenal inflammation with hepatic steatosis represent a distinction of pancreatitis in SARS-CoV-2.(26)

Pandanaboyana et al., showed those with SARS-CoV-2 and acute pancreatitis had a significantly increased risk of developing moderate to severe or even severe acute pancreatitis, and a higher risk of secondary complications. They also had higher mortality compared to the group without the virus infection. However, this finding may have been due to the more advanced age, worse functionality score, and more severe and advanced stages of acute pancreatitis. ${ }^{(31)}$ Dirweesh et al., also identified higher mortality in the SARS-CoV-2 infected group. In their cohort study, they diagnosed acute pancreatitis according to the Atlanta Classification, and found a higher incidence of multiple organ failure and persistent organ failure in this cohort. ${ }^{(24)}$ In their prospective study, Akarsu et al., also showed that acute pancreatitis in patients with COVID-19 may deteriorate their clinical status and increase mortality. ${ }^{(16)}$

Not only in the adult group, Suchman et al., performed a retrospective study of patients under 18 years of age admitted to twelve New York City hospitals, between March and June 2020. Thirteen patients in the study were diagnosed with acute pancreatitis by the International Study Group of Pediatric Pancreatitis: In Search for a CuRE (INSPIRRE) criteria, ten of whom were diagnosed with idiopathic pancreatitis, with only two COVID-19 positive and the remainder negative. The authors suggest acute pancreatitis may occur in pediatric patients, and may be more common in those infected with SARS-CoV-2, particularly if there are associated gastrointestinal symptoms. ${ }^{(14)}$

Regarding imaging findings, Shiralkar et al., analyzed abdominal and thoracic CT and MRI scans of patients admitted with COVID-19 to the service included in the study. Ninety percent of them had pulmonary findings typical of COVID-19, and $25 \%$ of them had gastrointestinal findings of intestinal wall, pancreatitis, and cholecystitis. Among these patients, $70 \%$ had gastrointestinal symptoms upon admission, and $30 \%$ had them throughout their hospitalization. The authors call for early testing for SARS-CoV-2 in patients with typical or even atypical gastrointestinal symptoms, since it can lead to earlier diagnosis and isolation. ${ }^{(30)}$ Along the same line, Gupta, in a metaanalysis, suggests associating clinical-radiological tests for the diagnosis of acute pancreatitis and COVID-19 and, furthermore, warns about exposure to peritoneal fluid, if a surgical approach is necessary, since this fluid or other peritoneal fluid may contain viral particles and be a source of contamination for the staff. ${ }^{(17)}$

Three of the studies analyzed demonstrated no relation between the virus and acute pancreatitis. Samanta et al., in five articles analyzed in systematic review, did not conclude about this relation. ${ }^{(29)}$ McGuinness et al., compared hospitalization for acute abdomen, and there was no significant difference between 2019 and 2020 in severity of disease in patients with acute appendicitis $(\mathrm{p}=0.970)$, acute diverticulitis $(\mathrm{p}=0.333)$, or acute pancreatitis $(\mathrm{p}=0.803)$. However, this study was carried out in New Zealand, where there were few cases of COVID-19 cases during the study period. ${ }^{(34)}$ Miró et al., found that hospital admission for acute pancreatitis as a presentation of COVID-19 is uncommon in emergency departments, and demonstrated that in-hospital mortality does not differ between patients with acute pancreatitis, with or without concomitant viral infection. However, mortality in COVID-19 patients was higher in the setting of acute pancreatitis, perhaps due to the severity of presenting both diseases at the same time. ${ }^{(20)}$

Regarding published case reports, Juhász et al., pointed out that not all of them follow the case report guidelines, often skipping steps in the investigation of acute pancreatitis or not giving importance to the effect of several drugs used in the treatment of COVID-19 at hospital settings, which have also been described as a cause of acute drug-induced pancreatitis. ${ }^{(18)}$

The 39 case reports included in this study (some with case series) totaled 42 patients. Not all studies presented complete data of the clinical case and diagnosis of acute pancreatitis.

Most studies diverge as to diagnostic criteria, with three reports based on the Atlanta Classification; three on the modified Atlanta Classification; two on the modified Glasgow coma scale for acute pancreatitis; 17 joining clinical, laboratory, and imaging data; seven only with abdominal computed tomography (CT); two with clinical diagnosis; five with clinical and laboratory data, and one with clinical data and abdominal CT. Among the signs of acute pancreatitis on imaging, in 33 of 42 cases there were descriptions of suggestive findings, such as pancreatic edema, blurring of peripancreatic 
fat, necrosis, pancreatic pseudocyst, among others. Only three studies presented cases of patients with a history of mild alcoholism ${ }^{(41)}$ and cholelithiasis. ${ }^{(64,71)}$

Among the characteristics of patients $(n=42)$, 19 had comorbidities, sometimes combined, such as obesity or overweight (8), hypertension (10), type 1 or 2 diabetes mellitus (six), and hypercholesterolemia (one). The female patients appeared subtly more numerous than males - 22 and 19 cases, respectively. The mean age was 46.64 years, with a minimum of 11 years and a maximum of 78 years, with a median of 47 years and a mode of 67 years (four patients), and 36 years (four patients). Only seven studies cited the continuous use of medications, that is, beta-blocker, valsartan, clopidogrel, acetylsalicylic acid (ASA), atorvastatin, trastuzumab, losartan, metformin, insulin, omeprazole, nifedipine, carvedilol, levothyroxine, alendronate, cyanocobalamin (Vitamina B12), and alprazolam.

Among the initial symptoms that led the patient to seek medical care, gastrointestinal $(64.28 \%)$, respiratory $(47.61 \%)$, and fever $(54.76 \%)$ problems were the most common. Regarding abdominal pain, it was typical in the epigastric region, in a band-like area, irradiating to the back (12/42); only epigastric (13/42); diffuse $(8 / 42)$; in the right iliac fossa $(1 / 42)$, periumbilical $(1 / 42)$, or absent (3/42); four studies did not mention abdominal pain.

The diagnosis of pneumonia was made in 34 patients, 16 of whom had SARS per se. Only one article did not mention the diagnosis of COVID-19, ${ }^{(39)}$ and, in the others, it was made by reverse transcriptase polymerase chain reaction (RT-PCR).

Regarding laboratory tests, 28 studies included serum amylase results, with a mean of $779.25 \mathrm{U} / \mathrm{L}$ (the highest being 3,568U/L); 32 included serum lipase, with a mean of $1,230.88 \mathrm{U} / \mathrm{L}$ (the highest being $8,352 \mathrm{U} / \mathrm{L}$ ); C-reactive protein was cited in 25 articles, with a mean of $9.73 \mathrm{mg} / \mathrm{dL}$ (the highest being $29 \mathrm{mg} / \mathrm{dL}$ ); and triglycerides were performed in 15 articles, with a mean of $191.54 \mathrm{mg} / \mathrm{dL}$ (the highest being $310 \mathrm{mg} / \mathrm{dL}$ )-nine articles did not cite the triglyceride result, but described it as normal.

The medications used during hospitalization were cited in 18 of the reports included in the study. There were antibiotics, antimalarials, antivirals, corticosteroids, anticoagulants, bronchodilators, zinc, vitamin D, pantoprazole, and ondansetrone.

Regarding the evolution of cases, the most frequent complications were the need for non-invasive ventilation (NIV) with some oxygen supplementation (11/42), followed by the need for orotracheal intubation (10/42). Other noteworthy complications were kidney failure and need for hemodialysis (5/42), use of extracorporeal membrane oxygenation (ECMO) (3/42), and cardiac arrest (3/42). Among other less frequent events in the sample were the need to conduct term $(1 / 42)$ and premature (1/42) labor, diabetic ketoacidosis $(1 / 42)$, pulmonary thromboembolism $(1 / 42)$, refractory distributive shock (1/42), acute appendicitis concomitant with pancreatitis (1/42), pancreatic pseudocyst and its drainage (1/42), and laparotomy approach due to extensive necrosis $(1 / 42)$. Two reports did not provide complications. The outcome of the cases was mostly resolution of the condition (34/42), with five deaths. Three reports did not provide the outcome.

\section{DISCUSSION}

The pancreas as an extrapulmonary site in SARS-CoV-2 infection is still a doubt among several researchers. An interesting number of case reports suggested this association, but there is no pattern among them, which makes analysis and comparison among studies difficult. In addition, there is a small number of studies that are not case reports, but in those found in this research, most of them concluded there is some association.

The hypothesis that the increasing diagnosis of acute idiopathic pancreatitis is related to COVID-19 as a possible infectious etiology, is due to the known ability of other viruses to infect and inflame the gland. ${ }^{(13)}$ Therefore, associating the two causes could be plausible. Furthermore, SARS-CoV-2 causes infection in cells by binding its glycoprotein (spike protein $\mathrm{S}$ ) to the ACE2 receptor, which is present in various tissues, including the pancreas. ${ }^{(4)}$ It is also known that ACE2 participates in the regulation of metabolism through its action in the gland, allowing better insulin secretion and glucose homeostasis. ${ }^{(73)}$ Interestingly, the expression of SARS-CoV-2 in the pancreatic tissue, in addition to supporting the hypothesis it causes acute pancreatitis, can also cause insulin-dependent diabetes mellitus by destroying the pancreatic islets. ${ }^{(74)}$ Additionally, several studies have demonstrated pancreatic involvement with histopathological analysis in autopsy of patients with COVID-19..$^{(9,10)}$

In contrast, another pathological study that also identified viral RNA in the pancreas analyzed the SARS-CoV-2 RT-PCR Ct (RT-PCR-cycle threshold) values. ${ }^{(11)}$ These values are used to measure the prognosis of the disease. Low values correlated with a greater risk of a serious evolution and higher mortality and, therefore, worse prognosis. ${ }^{(75)}$ In the study, higher $\mathrm{Ct}$ values were found in non-respiratory tissues, and no active viral replication or hybrid virus capture was identified in them. It was concluded that, although RTPCR for SARS-CoV-2 was positive in non-respiratory tissues, such as the pancreas, this finding might be due 
to residual viral RNA in the blood of these organs. The much-feared cytokine storm and multiple organ failure, which occur in severe forms of COVID-19, resemble the lipotoxicity process in severe acute pancreatitis. ${ }^{(15)}$ Thus, some authors suggested that in a patient in moderate to severe state, pancreatitis may be either by systemic inflammation of COVID-19 or by the virus itself in the pancreas. Severe cases of COVID-19 are strongly related to comorbidities, mainly hypertension, followed by diabetes mellitus, coronary disease, and obesity, among others. ${ }^{(3)}$ Of the 42 patients described in the included reports, 19 presented at least one of these diseases, which may support the theory of exacerbated systemic inflammation in response to the virus that caused the pancreatic injury, and not the direct viral action in the gland.

Acute pancreatitis is a multifactorial inflammatory disease of the pancreas, and the major concern is the progression to severe forms, with high morbidity and mortality. ${ }^{(76)}$

The diagnosis is made using the 2012 Atlanta Classification, which includes typical abdominal pain (acute and persistent epigastric pain, of strong intensity, irradiating to the back), serum lipase or serum amylase increased by at least three times the normal limit, and imaging findings compatible with acute pancreatitis on contrast-enhanced CT, MRI, or abdominal ultrasound. The diagnosis is established with at least two of the three criteria listed, and the disease is classified as mild, moderate, or severe, according to the patient's evolution within 48 hours. This criterion is accepted worldwide, leading to a consensus for the diagnosis of the disease, as well as better differentiation between the presentations (acute peripancreatic collection, pseudocyst, necrosis, and walled-off necrosis), and guidance for the most appropriate treatment. ${ }^{(77)}$

Among the case reports included in this study, only six explicitly stated that the Atlanta Classification was used, which makes it difficult to compare with the others that cited diagnosis only by imaging tests (seven studies) or clinical examination (one), because the universally established criteria were not respected, leading to hasty conclusions. Although 23 studies did not directly cite the Atlanta Classification, they respected the guideline by presenting at least two criteria, used other severity scales, such as Ranson, or modified Glasgow for acute pancreatitis, also accepted internationally. The diagnosis, in general, was well performed, with 31 studies against eight others without the Atlanta Classification. However, given the important systemic manifestations in SARS-CoV-2 infection, it is necessary to use the globally accepted criteria to properly validate the diagnosis of acute pancreatitis in a potentially severe patient. This is an important orientation, both to guide the management of disease, for statistical consideration, and for studies on viral infectivity in the pancreas, the characteristics of symptoms, and their evolution.

It is interesting to note there was a greater increase in lipase compared to amylase. Lipase is more sensitive than amylase for the diagnosis of acute pancreatitis, since it has a higher peak and stays elevated longer. However, lipase can be elevated for several reasons, such as cytotoxic effects of COVID-19 or increased intestinal permeability, as in critical illness in intense care unit (ICU), diabetes, use of opioids, and diarrhea. ${ }^{(66,78)}$

Several authors have noted divergences in diagnoses of acute pancreatitis, mainly because hyperlipasemia draws so much attention. Rasch et al., warned this finding is a result from impaired microcirculation rather than an extrapulmonary finding of viral infection per se. ${ }^{(22)}$ McNabb-Baltar et al., further demonstrated hyperlipasemia was not related to acute pancreatitis, since $48 \%$ of patients in the study with this finding did not present with laboratory or tomographic criteria to definitely make diagnosis. ${ }^{(19)}$ Along the same line, in his meta-analysis Gupta also alerted to the need of complementation of increased serum lipase with clinical and radiological findings. ${ }^{(17)}$ Wang et al., also reported increased pancreatic enzymes and associated this finding with pancreatic injury, but could not conclude whether there was a relation with direct viral damage to the gland. ${ }^{(35)}$ However, in this latter study, the classification of pancreatic injury did not follow the Atlanta Classification, with mean normal value or slightly elevated values of lipase and amylase - 77U/L and $86 \mathrm{U} / \mathrm{L}$, respectively.

Therefore, it is even more important to use the Atlanta Classification, since in the presence of SARS-CoV-2 infection, hyperlipasemia, or hyperamylasemia may occur, with or without clinical or radiological findings of acute pancreatitis. These findings should be supplemented with clinical and imaging examinations, to elucidate the diagnosis and adequately treat the patients.

On the other hand, three studies ${ }^{(28,32,33)}$ suggested hyperlipasemia can be used as a prognostic value in critically-ill patients with COVID-19. They demonstrated that higher values were associated with increased risk for poor outcomes, ICU admission, intubation, and mechanical ventilation time. Although McNabb-Baltar et al., ${ }^{(19)}$ concluded otherwise, these other three studies have relevant numbers of patients and similar results, which help to clarify the role of increased serum lipase in these cases. Thus, dosing the enzyme, especially upon admission of critically-ill patients, may contribute to more targeted care, as a simple and low-cost test that would act as a prognostic 
factor. Given the serious situation in several hospitals around the world, including Brazil, at the peak of the pandemic, with ICU capacity rates above $80 \%$ in several states, ${ }^{(79)}$ dosing of lipase could help in the allocation of patients and predict the care they may need, such as ICU admission and mechanical ventilation, improving hospital organization and patient management.

The fact that most patients described in the included reports sought medical care for gastrointestinal symptoms $(64.28 \%)$, typical or atypical of acute pancreatitis, is a worrisome fact. Most presented only with extrapulmonary symptoms, had respiratory symptoms late in their hospitalization, or presented suggestive tomographic findings by chance, such as ground-glass opacities, in the absence of respiratory symptoms. These facts have led teams to test RT-PCR for SARS-CoV-2 in these cases. Dietrich et al., described the case of a 72-year-old patient who presented with nausea and abdominal pain for 7 days. On investigation, an abdominal ultrasound showed cholelithiasis with no signs of cholestasis, and pancreatic parenchyma barely visible and apparently heterogeneous. Endoscopy and transesophageal ultrasound showed heterogeneous pancreatic tissue, with no focal masses, biliary duct with no signs of intraluminal or papillary calculi, suggesting acute non-biliary pancreatitis and no alcoholic etiology, since there was no past history of alcoholism. The patient progressively worsened until, on the fifth day of hospitalization, his chest CT scan showed bilateral ground-glass opacities. He was immediately isolated and subsequently confirmed with COVID-19.(71) Thus, it is important to reinforce the use of personal protective equipment, even when treating patients without classic symptoms of the virus, since this may be an extrapulmonary manifestation of the virus or an early presentation.

The gastrointestinal tract is a known focus of SARS-CoV-2. In March 2020, Tian et al., found a 3\% to $79 \%$ incidence of patients with gastrointestinal symptoms in SARS-CoV-2 infection, including anorexia (39.9\% to $50.2 \%$ ), diarrhea ( $2 \%$ to $49 \%$ ), vomiting (3.6\% to $66.7 \%$ ), nausea (1\% to $29.4 \%$ ), abdominal pain $(2.2 \%$ to $6 \%$ ), and gastrointestinal bleeding (4\% to $13.7 \%$ ). Diarrhea was the most common symptom in both adults and children, and was observed before and after diagnosis. More alarmingly, adults and children could present such symptoms without manifesting any respiratory complaints. ${ }^{(5)}$ These facts agree with those on table 2, in which most patients sought medical care without respiratory complaints, and were later diagnosed with the virus. ${ }^{(6,38,41-45,48,51,54,60,64,66,67,69,71)}$
The typical presentation of acute pancreatitis, with abdominal pain, nausea, and vomiting, fits into the most common set of gastrointestinal symptoms of COVID-19. Furthermore, diarrhea as an initial symptom was present in four of the reports included in this study, raising an alarm for the differential diagnosis of acute pancreatitis with SARS-CoV-2, or, in addition, alerting to the concomitant presence of these diseases, which, by causing intense systemic inflammation, increase morbidity and mortality in these patients. It is extremely important to apply the Atlanta Classification to diagnose acute pancreatitis and test for the virus, to identify these patients early, assist in their management, and protect the staff and other patients hospitalized at the same service for other causes.

Following the same reasoning, there are studies proving the presence of the virus in the peritoneal and peripancreatic fluid, and in pancreatic pseudocysts, sometimes in viral concentrations even higher than in the respiratory tract, poses a risk for infection of the staff during invasive procedures. ${ }^{(17,40,54,69)}$ Therefore, more protective measures should be taken if the surgical management of these patients is necessary.

The measures of care for acute pancreatitis initially include aggressive volume replacement and oral fasting, and in COVID-19, depending on the status of the patient, such measures would not be taken. ${ }^{(12)}$ In addition, some drugs can be toxic to the pancreas and even cause drug-induced pancreatitis, such as antivirals (lopinavir and ritonavir), antipyretics, tocilizumab, and baricitinib. ${ }^{(7)}$ In the case reports presented on table 2, only 18 of them cited drugs used during hospitalization - among them lopinavir (5/19), ritonavir (4/19), and tocilizumab (1/19); in four patients the concomitant use of lopinavir and ritonavir was used, and in one of them the combined use of these three drugs. Early detection of patients with acute pancreatitis and SARS-CoV-2 is necessary to avoid the occurrence of drug-induced pancreatitis, although rare, in patients already susceptible to severe systemic inflammation, or worsening of an already installed acute pancreatitis.

\section{CONCLUSION}

The literature is still divergent regarding pancreatic involvement in COVID-19. There are several confounding factors in the diagnosis of acute pancreatitis during concomitant infection by SARS-CoV-2, but the currently available information offers important warnings. First, in relation to the clinic, since in most cases the search for the health service was for 
abdominal pain, which should raise the suspicion of acute pancreatitis and other gastrointestinal diseases, as well as SARS-CoV-2 infection. Second, regarding the use of hyperlipasemia as a risk factor for admission to the intensive care unit, and use of mechanical ventilation. Third, the importance of following the Atlanta Classification or modified Atlanta Classification in patients with COVID-19 with increased pancreatic enzymes and/or gastrointestinal symptoms. Similarly, patients presenting with acute pancreatitis should be tested for COVID-19, since the clinical picture may be similar.

Thus, it is possible to identify early two diseases that can develop into serious and even fatal conditions, and to detect and isolate these patients more quickly. Additional studies are needed to prove the virus is capable of infecting the pancreas, but the data analyzed here are an important starting point.

\section{ACKNOWLEDGMENTS}

To Professor Dr. Ethel Zimberg Chehter, for assisting in search and preparation of this work.

\section{AUTHORS' CONTRIBUTION}

Júlia Aith Balthazar: contributed with the design and implementation of the research, to the analysis of the results and to the writing of the manuscript. Ethel Zimberg Chehter: was responsible for supervising the project and analyzing the results.

\section{AUTHORS' INFORMATION}

Balthazar JA: http://orcid.org/0000-0003-0643-7513

Chehter EZ: http://orcid.org/0000-0002-2409-5871

\section{REFERENCES}

1. Allen J, Almukhtar S, Aufrichtig A, Barnard A, Bloch M, Cahalan S, et al. Coronavirus World Map: tracking the global outbreak. New York: The New York Times; 2021 [Updated 2021 July 14, cited 2021 Apr 7]. Available from: https://www.nytimes.com/interactive/2021/world/covid-cases.html

2. Hu B, Guo H, Zhou P, Shi ZL. Characteristics of SARS-CoV-2 and COVID-19. Nat Rev Microbiol. 2021;19(3):141-54. Review.

3. Asselah T, Durantel D, Pasmant E, Lau G, Schinazi RF. COVID-19: discovery, diagnostics and drug development. J Hepatol. 2021;74(1):168-84. Review.

4. Saponaro F, Rutigliano G, Sestito S, Bandini L, Storti B, Bizzarri R, et al. ACE2 in the era of SARS-CoV-2: controversies and novel perspectives. Front Mol Biosci. 2020;7:588618. Review.

5. Tian Y, Rong L, Nian W, He Y. Review article: gastrointestinal features in COVID-19 and the possibility of faecal transmission. Aliment Pharmacol Ther. 2020:51(9):843-51. Review.
6. Miao Y, Lidove O, Mauhin W. First case of acute pancreatitis related to SARSCoV-2 infection. Br J Surg. 2020;107(8):e270.

7. Zippi M, Hong W, Traversa G, Maccioni F, De Biase D, Gallo C, et al Involvement of the exocrine pancreas during COVID-19 infection and possible pathogenetic hypothesis: a concise review. Infez Med. 2020;28(4):507-15. Review.

8. Liu F, Long X, Zhang B, Zhang W, Chen X, Zhang Z. ACE2 expression in pancreas may cause pancreatic damage after SARS-CoV-2 infection. Clin Gastroenterol Hepatol. 2020;18(9):2128-30.e2.

9. Yao XH, Li TY, He ZC, Ping YF, Liu HW, Yu SC, et al. [A pathological report of three COVID-19 cases by minimal invasive autopsies]. Zhonghua Bing Li Xue Za Zhi. 2020;49(5):411-7. Chinese.

10. Cîrstea $A E$, Buzulică RL, Pirici D, Ceauşu MC, Iman RV, Gheorghe OM, et al. Histopathological findings in the advanced natural evolution of the SARSCoV-2 infection. Rom J Morphol Embryol. 2020;61 (1):209-18.

11. Bhatnagar J, Gary J, Reagan-Steiner S, Estetter LB, Tong S, Tao Y, et al Evidence of SARS-CoV-2 replication and tropism in the lungs, airways and vascular endothelium of patients with fatal COVID-19: an autopsy caseseries. J Infect Dis. 2021;223(5):752-64.

12. Boxhoorn L, Voermans RP, Bouwense SA, Bruno MJ, Verdonk RC, Boermeester MA, et al. Acute pancreatitis. Lancet. 2020;396(10252):726-34. Review.

13. Rawla P, Bandaru SS, Vellipuram AR. Review of infectious etiology of acute pancreatitis. Gastroenterology Res. 2017;10(3):153-8. Review.

14. Suchman K, Raphael KL, Liu Y, Wee D, Trindade AJ; Northwell COVID-19 Research Consortium. Acute pancreatitis in children hospitalized with COVID-19. Pancreatology. 2021;21(1):31-3.

15. Hegyi P, Szakács Z, Sahin-Tóth M. Lipotoxicity and cytokine storm in severe acute pancreatitis and COVID-19. Gastroenterology. 2020;159(3):824-7.

16. Akarsu C, Karabulut M, Aydin H, Sahbaz NA, Dural AC, Yegul D, et al. Association between acute pancreatitis and COVID-19: could pancreatitis be the missing piece of the puzzle about increased mortality rates? J Invest Surg. 2020 Nov 2:1-7. doi: 10.1080/08941939.2020.1833263.

17. Gupta V. COVID-19 and acute pancreatitis: what do surgeons need to know? Indian J Surg. 2020 Jun 5:1-4. doi: 10.1007/s12262-020-02447-w. [Epub ahead of print].

18. Juhász MF, Ocskay K, Kiss S, Hegyi P, Párniczky A. Insufficient etiological workup of COVID-19-associated acute pancreatitis: a systematic review. World J Gastroenterol. 2020;26(40):6270-8. Review.

19. McNabb-Baltar J, Jin DX, Grover AS, Redd WD, Zhou JC, Hathorn KE, et al. Lipase elevation in patients with COVID-19. Am J Gastroenterol. 2020;115(8):1286-8.

20. Miró Ò, Llorens P, Jiménez S, Piñera P, Burillo-Putze G, Martín A, MartínSánchez FJ, Lamberechts J, Alquézar-Arbé A, Jacob J, Noceda J, Cano Cano MJ, Fortuny Bayarri MJ, Marín Porrino JM, Meléndez N, Pérez García C, Brasó Aznar JV, Ponce MC, Díaz Fernández E, Ejarque Martínez L, Peiró Gómez A, Tost J, Domínguez MJ, Teigell Muñoz FJ, González Del Castillo J; Spanish Investigators on Emergency Situations TeAm (SIESTA) network. A case-control emergency department-based analysis of acute pancreatitis in COVID-19: results of the UMC-19-S6. J Hepatobiliary Pancreat Sci. 2021;28(11):953-66.

21. Lax SF, Skok K, Zechner P, Kessler HH, Kaufmann N, Koelblinger C, et al. Pulmonary arterial thrombosis in COVID-19 with fatal outcome: results from a prospective, single-center, clinicopathologic case series. Ann Intern Med. 2020;173(5):350-61.

22. Rasch S, Herner A, Schmid RM, Huber W, Lahmer T. High lipasemia is frequent in COVID-19 associated acute respiratory distress syndrome. Pancreatology. 2021;21(1):306-11.

23. Inamdar S, Benias PC, Liu Y, Sejpal DV, Satapathy SK, Trindade AJ; Northwell COVID-19 research consortium. Prevalence, risk factors, and outcomes of hospitalized patients with coronavirus disease 2019 presenting as acute pancreatitis. Gastroenterology. 2020;159(6):2226-8.e2.

24. Dirweesh A, Li Y, Trikudanathan G, Mallery JS, Freeman ML, Amateau SK. Clinical outcomes of acute pancreatitis in patients with coronavirus disease 2019. Gastroenterology. 2020;159(5):1972-4. 
25. Hanley B, Naresh KN, Roufosse C, Nicholson AG, Weir J, Cooke GS, et al. Histopathological findings and viral tropism in UK patients with severe fatal COVID-19: a post-mortem study. Lancet Microbe. 2020;1(6):e245-e53.

26. Szatmary P, Arora A, Thomas Raraty MG, Joseph Dunne DF, Baron RD, Halloran $\mathrm{CM}$. Emerging phenotype of severe acute respiratory syndrome-coronavirus 2-associated pancreatitis. Gastroenterology. 2020;159(4):1551-4.

27. Gubatan J, Levitte S, Patel A, Balabanis T, Sharma A, Jones E, et al. Prevalence, risk factors and clinical outcomes of COVID-19 in patients with a history of pancreatitis in Northern California. Gut. 2021;70(2):440-1.

28. Akkus C, Yilmaz H, Mizrak S, Adibelli Z, Akdas O, Duran C. Development of pancreatic injuries in the course of COVID-19. Acta Gastroenterol Belg. 2020;83(4):585-92.

29. Samanta J, Gupta R, Singh MP, Patnaik I, Kumar A, Kochhar R. Coronavirus disease 2019 and the pancreas. Pancreatology. 2020;20(8):1567-75. Review.

30. Shiralkar K, Chinapuvvula N, Ocazionez D. Cross-sectional abdominal imaging findings in patients with COVID-19. Cureus. 2020;12(8):e9538.

31. Pandanaboyana S, Moir J, Leeds JS, Oppong K, Kanwar A, Marzouk A, Belgaumkar A, Gupta A, Siriwardena AK, Haque AR, Awan A, Balakrishnan A, Rawashdeh A, Ivanov B, Parmar C, M Halloran C, Caruana C, Borg CM, Gomez D, Damaskos D, Karavias D, Finch G, Ebied H, K Pine J, R A Skipworth J, Milburn J, Latif J, Ratnam Apollos J, El Kafsi J, Windsor JA, Roberts K, Wang K, Ravi K, V Coats M, Hollyman M, Phillips M, Okocha M, Sj Wilson M, A Ameer N, Kumar N, Shah N, Lapolla P, Magee C, Al-Sarireh B, Lunevicius R, Benhmida R, Singhal R, Balachandra S, Demirli Atıcı S, Jaunoo S, Dwerryhouse S, Boyce T, Charalampakis V, Kanakala V, Abbas Z, Nayar M; COVID PAN collaborative group. SARS-CoV-2 infection in acute pancreatitis increases disease severity and 30-day mortality: COVID PAN collaborative study. Gut. 2021;70(6):1061-9

32. Goyal H, Sachdeva S, Perisetti A, Mann R, Inamdar S, Tharian B. Hyperlipasemia and potential pancreatic injury patterns in COVID-19: a marker of severity or innocent bystander? Gastroenterology. 2021;160(3):946-8.e2.

33. Rathi S, Sharma A, Patnaik I, Gupta R. Hyperlipasemia in COVID-19: statistical significance vs clinical relevance. Clin Transl Gastroenterol. 2020;11(12):e00261.

34. McGuinness MJ, Harmston C. The effect of national public health interventions for COVID-19 on emergency general surgery in Northland, New Zealand. ANZ J Surg. 2021;91(3):329-34.

35. Wang $F$, Wang $H$, Fan J, Zhang $Y$, Wang $H$, Zhao 0 . Pancreatic injury patterns in patients with coronavirus disease 19 pneumonia. Gastroenterology. 2020;159(1):367-70

36. Aloysius MM, Thatti A, Gupta A, Sharma N, Bansal P, Goyal H. COVID-19 presenting as acute pancreatitis. Pancreatology. 2020;20(5):1026-7.

37. Anand ER, Major C, Pickering 0, Nelson M. Acute pancreatitis in a COVID-19 patient. Br J Surg. 2020;107(7):e182.

38. Hadi A, Werge M, Kristiansen KT, Pedersen UG, Karstensen JG, Novovic $S$, et al. Coronavirus disease-19 (COVID-19) associated with severe acute pancreatitis: case report on three family members. Pancreatology. 2020;20(4):665-7.

39. Meireles PA, Bessa F, Gaspar P, Parreira I, Silva VD, Mota C, et al. Acalculous acute pancreatitis in a COVID-19 patient. Eur J Case Rep Intern Med. 2020;7(6):001710.

40. Shinohara T, Otani A, Yamashita M, Wakimoto Y, Jubishi D, Okamoto K, et al. Acute pancreatitis during COVID-19 pneumonia. Pancreas. 2020;49(10):e106-8.

41. Meyers MH, Main MJ, Orr JK, Obstein KL. A case of COVID-19-induced acute pancreatitis. Pancreas. 2020;49(10):e108-9.

42. Cheung $S$, Delgado Fuentes $A$, Fetterman $A D$. Recurrent acute pancreatitis in a patient with COVID-19 infection. Am J Case Rep. 2020;21:e927076.

43. Kandasamy S. An unusual presentation of COVID-19: acute pancreatitis. Ann Hepatobiliary Pancreat Surg. 2020;24(4):539-41.

44. Lakshmanan S, Malik A. Acute pancreatitis in mild COVID-19 infection. Cureus. 2020;12(8):e9886.

45. Gonzalo-Voltas A, Uxia Fernández-Pérez-Torres C, Baena-Díez JM. Acute pancreatitis in a patient with COVID-19 infection. Med Clin (Engl Ed). 2020;155(4):183-4.
46. Brikman S, Denysova V, Menzal H, Dori G. Acute pancreatitis in a 61-year-old man with COVID-19. CMAJ. 2020;192(30):E858-9.

47. Narang K, Szymanski LM, Kane SV, Rose CH. Acute pancreatitis in a pregnant patient with coronavirus disease 2019 (COVID-19). Obstet Gynecol. 2021:137(3):431-3

48. Wang K, Luo J, Tan F, Liu J, Ni Z, Liu D, et al. Acute pancreatitis as the initia manifestation in 2 cases of COVID-19 in Wuhan, China. Open Forum Infect Dis. 2020;7(9):ofaa324.

49. Tollard C, Champenois V, Delemer B, Carsin-Vu A, Barraud S. An inaugura diabetic ketoacidosis with acute pancreatitis during COVID-19. Acta Diabetol. 2021:58(3):389-91.

50. Acherjya GK, Rahman MM, Islam MT, Alam AS, Tarafder K, Rahman MM, et al. Acute pancreatitis in a COVID-19 patient: an unusual presentation. Clin Case Rep. 2020;8(12):3400-7.

51. Karimzadeh S, Manzuri A, Ebrahimi M, Huy NT. COVID-19 presenting as acute pancreatitis: lessons from a patient in Iran. Pancreatology. 2020;20(5):1024-5.

52. Simou EM, Louardi M, Khaoury I, Abidi MA, Mansour A, Louadghiri AE, et al. Coronavirus disease-19 (COVID-19) associated with acute pancreatitis: case report. Pan Afr Med J. 2020;37:150.

53. Mazrouei SS, Saeed GA, Al Helali AA. COVID-19-associated acute pancreatitis: a rare cause of acute abdomen. Radiol Case Rep. 2020;15(9):1601-3.

54. Kumaran NK, Karmakar BK, Taylor OM. Coronavirus disease-19 (COVID-19) associated with acute necrotising pancreatitis (ANP). BMJ Case Rep. 2020;13(9):e237903

55. Kataria S, Sharif A, Ur Rehman A, Ahmed Z, Hanan A. COVID-19 induced acute pancreatitis: a case report and literature review. Cureus. 2020;12(7):e9169.

56. AlHarmi RA, Fateel T, Sayed Adnan J, AlAwadhi K. Acute pancreatitis in a patient with COVID-19. BMJ Case Rep. 2021;14(2):e239656.

57. Alwaeli H, Shabbir M, Khamissi Sobi M, Alwaeli K. A case of severe acute pancreatitis secondary to COVID-19 infection in a 30 -year-old male patient. Cureus. 2020;12(11):e11718.

58. Alves AM, Yvamoto EY, Marzinotto MA, Teixeira AC, Carrilho FJ. SARS-CoV-2 leading to acute pancreatitis: an unusual presentation. Braz J Infect Dis. 2020;24(6):561-4.

59. Fernandes DA, Yumioka AS, Filho HR. SARS-CoV-2 and acute pancreatitis: a new etiological agent? Rev Esp Enferm Dig. 2020;112(11):890.

60. Purayil N, Sirajudeen J, Va N, Mathew J. COVID-19 presenting as acute abdominal pain: a case report. Cureus. 2020;12(8):e9659.

61. Patnaik RN, Gogia A, Kakar A. Acute pancreatic injury induced by COVID-19. IDCases. 2020:22:e00959.

62. Rabice SR, Altshuler PC, Bovet C, Sullivan C, Gagnon AJ. COVID-19 infection presenting as pancreatitis in a pregnant woman: a case report. Case Rep Womens Health. 2020;27:e00228.

63. Bokhari SM, Mahmood F. Case report: Novel coronavirus-a potential cause of acute pancreatitis? Am J Trop Med Hyg. 2020;103(3):1154-5.

64. Samies NL, Yarbrough A, Boppana S. Pancreatitis in pediatric patients with COVID-19. J Pediatric Infect Dis Soc. 2021;10(1):57-9.

65. Kurihara Y, Maruhashi T, Wada T, Osada M, Oi M, Yamaoka K, et al Pancreatitis in a patient with severe coronavirus disease pneumonia treated with veno-venous extracorporeal membrane oxygenation. Intern Med. 2020;59(22):2903-6.

66. Bineshfar N, Mirahmadi A, Karbasian F, Pourbakhtyaran E, Karimi A, Sarafi M. Acute pancreatitis as a possible unusual manifestation of COVID-19 in children. Case Rep Pediatr. 2021:6616211.

67. Hassani AH, Beheshti A, Almasi F, Ketabi Moghaddam P, Azizi M, Shahrokh S. Unusual gastrointestinal manifestations of COVID-19: two case reports. Gastroenterol Hepatol Bed Bench. 2020;13(4):410-4.

68. Bouali M, Ouchane M, Elbakouri A, Bensardi F, Elhattabi K, Fadil A. Total gastric necrosis following acute pancreatitis in a patient with COVID -19: case report and literature review. Ann Med Surg (Lond). 2021;62:362-4. 
69. Schepis T, Larghi A, Papa A, Miele F, Panzuto F, De Biase L, et al. SARSCoV2 RNA detection in a pancreatic pseudocyst sample. Pancreatology. 2020;20(5):1011-2.

70. Ahmed AO, Mohamed SF, Saleh AO, Al-Shokri SD, Ahmed K, Mohamed MF. Acute abdomen -like-presentation associated with SARS-CoV-2 infection. IDCases. 2020;21:e00895.

71. Dietrich CG, Hübner D, Marx G, Bickenbach J, Bootsveld A. Primary presentation of COVID-19 solely with gastrointestinal symptoms: a problem for the containment of the disease. Eur J Gastroenterol Hepatol. 2020;32(11):1475-8.

72. Chivato Martín-Falquina I, García-Morán S, Jiménez Moreno MA. Acute pancreatitis in SARS-CoV-2 infection. Beyond respiratory distress. Rev Esp Enferm Dig. 2021;113(5):388-9.

73. Yuan L, Li Y, Li G, Song Y, Gong X. Ang (1-7) treatment attenuates $\beta$-cell dysfunction by improving pancreatic microcirculation in a rat model of Type 2 diabetes. J Endocrinol Invest. 2013;36(11):931-7.

74. Yang JK, Lin SS, Ji XJ, Guo LM. Binding of SARS coronavirus to its receptor damages islets and causes acute diabetes. Acta Diabetol. 2010;47(3):193-9.
75. Rao SN, Manissero D, Steele VR, Pareja J. A narrative systematic review of the clinical utility of cycle threshold values in the context of COVID-19. Infect Dis Ther. 2020;9(3):573-86. Review. Erratum in: Infect Dis Ther. 2020;9(3):573-86.

76. Zheng Z, Ding YX, Qu YX, Cao F, Li F. A narrative review of acute pancreatitis and its diagnosis, pathogenetic mechanism, and management. Ann Transl Med. 2021;9(1):69. Review.

77. Banks PA, Bollen TL, Dervenis C, Gooszen HG, Johnson CD, Sarr MG, Tsiotos GG, Vege SS; Acute Pancreatitis Classification Working Group. Classification of acute pancreatitis--2012: revision of the Atlanta classification and definitions by international consensus. Gut. 2013;62(1):102-11.

78. Ashok A, Faghih $M$, Singh VK. Mild pancreatic enzyme elevations in COVID-19 pneumonia: synonymous with injury or noise? Gastroenterology. 2021;160(5):1872.

79. Souza P, Galzo W. Ocupação de leitos de UTI ultrapassa 80\% em 23 estados e no DF. CNN Brasil; 2021 [atualizado 2021 Mar 12, citado 2021 Abr 7]. Disponível em: https://www.cnnbrasil.com.br/saude/2021/03/12/ocupacaode-leitos-de-uti-ultrapassa-80-em-23-estados-e-no-df 